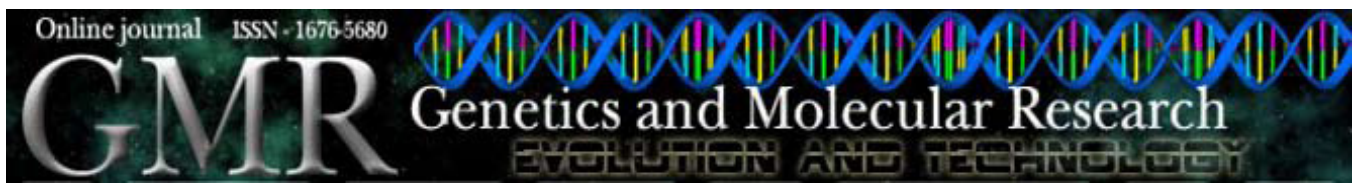

New Theory

\title{
A genomic parasite in the evolution of metazoan development
}

\author{
H.F. Hoenigsberg* and C. Sanabria \\ Instituto de Genética Evolutiva and Biología Molecular and \\ Instituto de Genética Ecológica y Biodiversidad del Trópico Americano, \\ Bogotá D.C., Colombia \\ Corresponding author: H.F. Hoenigsberg \\ E-mail: hoenigsberg@cable.net.co \\ *The first author dedicates this article to the memory of his mentors \\ Professors H.J. Muller (PN), Th. Dobzhansky, A. Buzzati-Traverso, and \\ Ruggero Ceppellini.
}

Genet. Mol. Res. 8 (3): 896-914 (2009)

Received December 15, 2008

Accepted June 28, 2009

Published July 28, 2009

ABSTRACT. It is a challenge to understand how development emerged
as a mechanism to dismantle and dismiss the intromission of foreign
parasites in order to consolidate a higher-level multicellular unit
of selection where more heritable variations in fitness, required for
complex organization, can be procured. Levels in biological hierarchy
genes, networks of genes, chromosomes, cells, organisms, etc., possess
heritable variations in fitness to varying degrees, and as such, they
function as units of selection in the evolutionary process [Lewontin,
(1970). The units of selection. Annu. Rev. Ecol. Syst. 1: 1-18]. To proceed
from each of these levels to the next constitutes a major transition in
evolutionary history. When analyzing the splendid road epitomized by
these transitions in units of selection, it is possible to conceive three
processes: firstly, the molecular "recognition" of the "convenience" of
exchanging the higher energy cost of cooperating cells with more fitness
than single-cell selection (after that first recognition the emergence of 
cooperation among cells is possible); secondly, the establishment of the mechanisms to regulate conflict, and finally, the regulation of cell differentiation and compartmentalization.

Key words: Molecular biology; Population genetics; Evolutionary mechanism; Metazoan development

\section{INTRODUCTION}

The system of information proposed by Eigen and Schuster (1979) had to contain only a few ( $\mathrm{v}_{\max } \sim<100$ nucleotides) units: but there had to be several such units with similar functions to build up a translational system. In order for these hypercycles to function, there had to exist several subpopulations (quasi-species in Eigen and Schuster's terminology) of them. Analogous partners cannot bond among themselves since this leads to fierce competition among the equivalent auto-catalytic and self-reproductive units within the compartments.

For the next functional step, linkage among self-reproductive units is required. The following conditions have to be fulfilled:

1. A first-degree cyclic linkage, which permits control of the relentless Darwinian kind of competition among self-reproductive units in order to retain resilient information.

2. Once present, the hypercyclic linkage must be able to control contentious first-order reproductive units.

3. To "jump" to the cooperative (among genes) mode, the transition level linkage must "switch off" or rather selectively depart from the competitive first-level unit to comply with some integrated new functional system.

4. In order to reach functional stability, and before competing as an integrated unit of selection, the bond of cooperating genes in a larger self-replicating unit has to retain conflict modifying codes in chromosome-linked structures. Although it is a very difficult and magnificent step that could have taken a long time to consolidate for metazoan development, this intermediate transition (not present in Eigen and Schuster's (1979) theory) appears indispensable to stabilize a gene system against the accumulation of errors just before molecular parasites or mutants ready to defect appear in the transitional scenario.

5. The integrated higher functional system must be able to compete favorably with other less efficient systems.

\section{CONTROL AND REGULATION OF CONFLICTS}

To materialize evolution gradually but in a progressive way, replicators have to assemble as groups of cooperating genes (Maynard Smith and Szathmáry, 1995; Hoenigsberg, 2003a,b). After that first unit of real Darwinian selection comes into being, other units of selection progressively follow up on adaptations in the form of cellular compartments, chromosomes, organisms, populations, species, and communities. Investigators of the origin of life, however, generally agree that its initiation did not enjoy the blessings of heredity, multiplication and variability (Eigen, 1971; Corliss et al., 1981; Gánti, 1971, 1984a,b; Hoenigsberg, 2007). Indeed, the dynamic aspect of life is best understood by Gánti's fluid machines whose workings are not constrained by the geometry of solid state structures (Griesemer, 2003). 
However, when replicators emerged from chemistry and heredity, selection and its product adaptation, guided gradual and progressive evolution by adding fitness values to the previous transition (Hoenigsberg, 2007). In this journey towards fitness, differential viability and fertility "showed" the way.

In order to further materialize progressive evolution, the next step in the mechanism had to include a certain type of group selection. Is this principle possible in population genetics theory? We have to cope with the various mechanisms and their functional devises (generally ignored by biologists) that construct Darwinian selection and adaptation (Williams, 1966). We do not have the necessary length in this paper to present all the pros and cons of the reality of group selection; all we can do at this time is to offer Sewall Wright's $(1945,1978)$ proposal for a certain group-selection's effectiveness in a species.

Our theory pertains as well to the entrance of parasites into a recently evolved multicellular choanoflagellate that did not initiate metazoan development.

On the heritable variations among close relatives (clones in our theory), the view of Wright (1945) is that group selection would be particularly effective in populations (especially so for multicellular animals derived from choanoflagellates) that are almost but not quite isolated from each other. Most of the evolutionary change in such a mosaic of small populations would be adjuvant and accrued periodically with allelic selection coefficients. However, the populations would be so small that the genes would occasionally be fixed by drift in spite of "jumps" of adverse selection within the population. Some of the fixed genes might benefit the population (augmenting cooperation among molecular dominions, for example) as a whole, even though they would be of competitive disadvantage within the population. Especially, Williams (1966) maintains that a group (of small populations) would increase in size (therefore in fitness) and permit an augmented number of emigrants to neighboring populations. Such migrants would repeatedly counteract the adverse selection of the gene (of some alleles in the small pool) in the neighboring populations and give them repeated opportunity (over and over again) for the chance fixation of the gene. The mathematical machinery created in this process (for bottlenecks, see Rodriguez and Hoenigsberg, 1987) can produce adaptation of group benefit, even though there would be a competitive disadvantage to an individual. Wright's escalating drift can also result from shrinking heterozygosis after population bottlenecks (Hoenigsberg and Rodriguez, 1987) and such population phenomena probably occurred frequently in the stages of the transition to multicellularity (King, 2004).

The control of conflicts is a prerequisite of the principles that guide the transition from molecular replicators (single genes) to groups and constitutes the mechanics for the creation of new units of selection and therefore of new fitness. Without genes which in their interaction with other genes intervene in the creation of higher-order information which modifies conflict, eases transition between levels and shifts fitness to deal with the higher cost demanded by cooperation, parasitic entrance into relatively well-established genomes would not have been overwhelmingly aimed at benefiting higher-order regulation. The control and regulation of conflict is central to promoting cooperation within the genome, the chromosome and the cytoplasmic inclusions, the cells that have to differentiate and the signals that are needed to produce functional phenotypes. However, conflict modifiers are also needed to encourage cooperation through selection in order to compensate for damage from lower-level units in the functioning of the group of cells. At the same time, the persistent confrontation of mutants or parasites serves to mitigate and discourage the tendency, not only of the lower-unit replicators 
but also of parasites, to drag down fitness (Hoenigsberg et al., 2008), resulting in the elimination or delaying of fixation of the appropriate alleles of the implicated gene pools. Since cooperation means complex organization and greater expenditure of energy, there is always the temptation among defectors and cheaters (mutants), to drag organisms down to a lesser expenditure of energy and therefore to a more chaotic Darwinian struggle. How might conflict destroy cooperation? The Darwinian machine for small populations had to act constantly to effect the appropriate change.

Therefore, cooperation and altruism can emerge in multicellular individuals. Although most theoretical biologists are consensual in defining cooperation, there is some degree of confusion concerning altruism (Kerr et al., 2004).

If we are to offer a theory that seeks to explain that development in the Metazoa evolved as the result of invasive parasitic cycles, which provoked a succession of multiple evolutionary crises quite similar to the transitions in the units of selection, we have to demonstrate that going from one developmental stage to the next involved changes in fitness values arising from the emergence of new units of selection for gradually building genes that cooperate.

\section{RELICS OF PARASITES ARE FOUND EVERYWHERE IN THE GENOME}

Doolittle and Sapienza (1980), amazed by the huge uninformative gene regions, wrote that these regions were indeed replete with selfish sequences. In the same year, Orgel and Crick (1980) wrote a theoretical paper on selfish DNA, the ultimate parasite.

Genes are just stretches of DNA that, under natural selection, end up coding for proteins. However, about $90 \%$ of our genome do not consist of coding genes (Watson et al., 2004). The genome, of probably most eukaryotes, consists of a huge assortment of sequences that do not seem to have specific constructive roles, and have received, as entities, names such as jumping genes, pseudogenes, retropseudogenes, transposons, retrotransposons, minisatellites, microsatellites, etc., collectively known as "junk" or in most cases, selfish DNA. Although some are genes of a special category, most are large or small sequences of nucleic acids that are never transcribed into RNA. Actually some selfish genes, in competing with other genes, end up with a selective value by virtue of their "mischievous" behavior in detriment to real genes. In Drosophila, segregation distorters actually violate the most important law of cell division: to carefully separate into equal parts the genetic endowment of the previous generation. The so-called selfish genes act on their own and promote themselves. They seem to constitute the fossil evidence of the first sequences of information from about 4000 million years ago, when the evolutionary process was just a Darwinian competition among single entities. Try to imagine the scenario where genomes of the size of 3000 megabases (some metazoans) were attempting to out complete each other. Even with just 4 megabases (as in a prokaryote) competing, hereditable individuals without the evolution of cooperation and altruistic behavior would result in a staggering enterprise.

\section{THE THEORY}

The entrance of a parasite into a multicellular metazoan of recent bilaterian origin in Precambrian days can be taken as an unexpected illegitimate sexual exchange. In contrast, our theory demands that the mitotic cycles from the first unicellular metazoan produced a high 
degree of consanguineous products. Such clones would not have genetic conflict with their parents because they were descendents of the same genome.

Single-celled eukaryotes, with their specialized locomotion, feeding and capacity for indefinite cell division, have a very limited control over their environment. However, the first multicellularity already represented a higher physiological fitness. This next step in the transition to multicellular Metazoa took place only after two great cellular revolutions: 1) the acquisition of a non-rigid metazoan membrane and 2) several revolutionary experiments to construct a cytoskeleton. Obviously, many other revolutionary changes occurred in the unicellular Vendian eukaryote. The endomembrane system of the eukaryotes consists of many different kinds of membranes: with ribosomes attached not to the cell membrane as in prokaryotes, but, in other species, to the endoplasmic reticulum, vacuoles and other internal structures to aid internal digestion, some eukaryotes have suspended in their cytoplasm an enormous amount of proteins involved in a cascade of intermediate signaling processes, etc. We can be certain of the progressive and gradual population genetic changes that occurred, because, while one group of prokaryotes have a very rigid cell wall, another, the so-called Archae-bacteria, which lack a cell wall, have instead a very rigid type of cell membrane. Moreover, evolutionists universally concede that evolving such a great deal of independence over their environment implies an outstanding amount of gradual selection and adaptive steps in metazoans. The cells of multicellular metazoans could largely produce their own intercellular conditions. However, there are constraints on metazoan ontogeny, as in their highly differentiated cells that are often characterized by a limited capacity for cell division.

Our theory is that the parasite, which entered and "inseminated" the metazoan host, found a local cascade of molecular reactions that led to development. We assert that many seminal injections were responsible for the many developmental exploits that produced the metazoan. It is generally contended that Vendian and early Cambrian biological explosions of body plans, from which monophyletic metazoan phyla diverged (Zrzavy et al., 1998; Adoutte et al., 2000; Medina et al., 2001), can be attributed to the enormous environmental changes attributed to extended periods of new physical conditions and atmospheric oxygen levels (reaching present levels). The shared features in different phyla, we believe, have shown an incipient common body plan (including paraphyly of sponges and demosponges; see Bavestrello et al., 1998; Borchiellini et al., 2001), which probably emerged when multicellularity arose some 650-750 million years ago (Conway Morris, 1993). Cell behavior, when our first parasite entered, was not simply the direct response to the surrounding environment (as in singlecelled eukaryotes). Multicellular metazoans at that time already resorted to an advanced and sophisticated system of cell behavior in which they largely produced their own intercellular conditions. Cell behavior in the multicellular metazoan-candidate-for-development emerged responsive to these interactions; the multicellular metazoan was already capable of creating its own conditions (Erwin and Davidson, 2002).

\section{UNEQUAL INHERITANCE OF CELL FATE DETERMINANTS}

When parasitic invasions provoked intercellular conditions (through transcription factors), the first metazoan that began responding with a higher level of selection found its organic adaptation in cell differentiation (cell types) and compartmentalization. Submitting their genome to a higher level of selection and adaptation, the reward was by way of coopera- 
tion among genes, instead of the competitive lower-level selection. Since homologues of Hox genes in plesiomorphic characters in arthropods and vertebrates are evidence of non-adaptations in today's environments, it is likely that genes utilized by the new reactant-machinery of the host spread by catapulting the cooperative effects by virtue of their surviving merits.

\section{PARASITES INITIATED A MAJOR EVOLUTIONARY TRANSITION}

Cell differentiation already existed in the multicellular Metazoa as a secondary expression of the internal cellular conditions characteristic of them. Differentiation is ultimately a direct consequence of proteins, which in turn are the agents of cell function. Proteins that act as master regulators of genes determine individual cell types or even a whole group of cells. In the zygote, asymmetric cell divisions produce two different cells, one with a master regulator and the other without it. Thus, the multicellular Metazoa at the time of the proposed invasion of a foreign genome through a parasite already had an asymmetric distribution of molecules in their cytoplasm. In the cytoplasm, there are particles of mRNA, which act as master regulators in one region but not in another, as occurs in today's eggs. This early segregation of cytoplasmic particles and therefore of regulatory proteins in the female egg could be considered the origin of cell differentiation. Early segregation of master regulators through protein earlytarget specialization is the first fingerprint of selection to secure harmonious interactions. The zygote, protected by armoring, is constrained from the start and responds by blocking any early attempt at invasion. The parasites that enter the differentiated cell face an initial difficulty when exercising an extractive appropriation of host signals in the developmental genome. The RNA master regulator messenger in the zygote has no compatibility with the invasive genome! How did this cytoplasmic segregation come about from the selective mechanism?

The most parsimonious way was to produce asymmetric divisions by establishing a new and revolutionary axis of polarity. In other words, to orient the mitotic spindle along this axis and localize cell fate determinants to one side of the cell meant new cell types. In order for selection to orient metazoan cytoplasmic compartmentalization as an early instrument of protection against selfish molecular invasion, there had to exist a high variability in the incompatibility between the zygote $\mathrm{G}$ master regulator and invasive genomes. When selection occurs on several levels simultaneously, as often happened in the course of evolution, fitness at any one level is a poor guide to understanding the outcome. Average individual fitness is also of limited use in understanding, for example, frequency-dependent selection or the evolution of cooperation among cells in the transition from single cells to the multicellular metazoan.

When reproduction is sexual, an organism's offspring constitute a mixture of different genotypic products which, from its inception, include the contributions of gametes to other genotypic classes (Dobzhansky, 1970; Wright, 1978; Williams, 1966, 1992).

Trading increments in fitness in a higher level of selection for development, through cell differentiation and compartmentalization, is expensive for individuals yet beneficial to groups of highly related cells (Michod, 1999a; Hoenigsberg et al., 2008). Developmental cooperation in the multicellular Metazoa includes distinguishing various cell types with different properties that are determined by their specific gene activity and the proteins they produce. The pattern of gene expression in the nucleus of a differentiated cell can be reversed, implying that it has, to a great extent, machinery integrated by devices supplied by the cytoplasm; the genes never acquire the status of irreversible codes. Nuclear reversibility came about as 
a selective mechanism in the interface between the single and the multicellular status, when the complex molecular reactions of incipient cell differentiation had to respond to external invasions. This nuclear plasticity was crucial to the process, by responding to new invasive genomes with an evolvable and practical mechanism in the form of codes that could outdo the parasite. We assert that this stage is the trade off for fitness. A rigid genome cannot produce an immune reaction with resourcefulness. Nuclear plasticity with "resolve" can respond by dissipating lethal or damaging cytological effects. Moreover, instances of nuclear reversibility in various moments of development are indicators of the challenges met by the intercellular communication system as it destroys or ignores return signals of dubious origin that were not specific enough to be detected by the membrane receptor apparatus of the host. To confront invasions at different moments of metazoan development, cooperation among intercellular communications, aimed at coping with the molecular "army" of parasitic genomes sending chemical signals to nearby cells to control metazoan metabolism for their own reproductive machinery, was the only mechanism on hand to address infection. Then as now, the more sophisticated cooperative kin selection (among clones) had to control insupportable mutations that might endanger the survival of the host genome, or cope with the amiable hybrid (parasite/ host genome), which responds with an "anarchically" selfish behavior. Such a dangerous array of selfish codes, which are never accommodating to the host genome, could only be confronted by conflict modifiers at the cellular level or with policing mechanisms, such as mitotic metaphases, which rapidly detect inassimilable codes, certainly before they order transcription factors (master regulators).

\section{TRADING FITNESS AT DIFFERENT LEVELS IS THE RESULT OF ORGANIC ADAPTATION}

Then as now, the entrance of a foreign genome had an important task: to overcome the defenses of the host's multicellular genome in order to: 1) disrupt the already established level of cell cooperation by increasing intracellular change (through mutation); 2) disrupt intracellular compartments; 3) obliterate protein signals for genetic translation; 4) dismantle signals and target molecules necessary for the regional coherence of cell differentiation: 5) reduce intra- and intercellular cooperation to impotence, which is the same as saying, that it would try to increase change within the organism. In a few words, that is the minimum work the parasite must perform in order to appropriate the internal machinery in the midst of chaos. In a rush to survival, a unit of selection must possess heritable variations in fitness if adaptations are to evolve at this level of organization (Darwin, 1859). Therefore, to understand the origin of individuality and of evolvability, we must understand how the properties of heritability and fitness variation rise to a new level, higher and more durable, starting from the lower-level unit. Between two levels of selection that are facing individual death and extinction the most convenient result is increasing the allelic frequency of those genes in the gene pool that best respond with harmonious cooperation among the cells.

\section{TO ACCEDE TO HIGHER LEVELS OF SELECTION, INTERCELLULAR SIGNALING HAD TO BE PERFECTED}

The multicellular metazoans arose, some time in the early Vendian, as unicellular or- 
ganisms that enjoyed a long evolutionary history before they merged to produce multicellularity (Erwin, 1999). Although some uncertainties persist, especially over the position of nematodes, we have based our studies of metazoan phylogeny on 18S rDNA (Halanych et al., 1995; Aguinaldo et al., 1997). A proterostome-deuterostome ancestor (PDA) close to the amazing evolutionary burst could indicate a greater role for developmental innovation (Erwin, 1999). Indeed molecular evidence indicates a maximum age of about $670 \mathrm{Ma}$. Our ultimate bewildering question turns out to be, why is such a revolutionary innovation clustered in that particular period of time? Could it be that morphological innovations derive from the constraints created by the new developmental programs? Couldn't widely accepted ecological opportunities of the Vendian be the originators of our proposed parasitic susceptibility of those well-equipped unicellular metazoans that were the parental generation?

Every now and then, directed evolution strategies appear to constitute the most efficient method for creating proteins with improved or novel properties. Directed evolution strategies are what you do to accede to a higher level of selection. To do that, individual cells relinquish their previous evolutionary heritage in favor of the organism. Selection and interaction among cells, below the level of the organism, destroy the harmony within the organism. Competition, which in the unicellular condition is optimal for Darwinian selection, now has to be avoided, since it is likely to favor defecting cells that pursue their own interests at the expense of the organism.

Competition can be avoided when selection operates in a mosaic of small populations (Wright, 1945). And, under variable environmental conditions, cells had to create proteins with improved properties and be more variable in their structure. Competition could be an obstacle for such immediate problem. The genetic solution to facing survival in the midst of continuous environmental changes is de novo library synthesis to allow for a true rational design that is based on available information about maximal protein flexibility. After all, if ecological conditions that cannot predict from the parental gene pool because of continuous environmental changes are an impediment for such predictions, the best solution is to produce variable genotypes with alleles capable of coding proteins with variable competences.

To create de novo library synthesis must have been quite an ordeal! In fact thousands must have found death at the end of the cascade of ...first cooperation and altruism ... or just in between.... and maybe on some rare occasions gradually a new protein with the right dominion for the best condition.

Therefore, since the entrance of the foreign genome implies a disruption of cooperation through induced mutations in the conflict-modifying "caucus", for the organism to reach the status of a new evolutionary unit, ways must be found to regulate or suppress a return to the selfish tendencies of cells. Thus, within-organism change and interaction must be controlled so that heritability of fitness can progressively increase at the organismic level under a mosaic of demic selection aided by drift.

Others argue (Maynard Smith and Szathmáry, 1995) that a close kinship among cells alone should be sufficient to regulate selfish tendencies of cells. It is by fusing meiotic cells in zygote formation (a single-cell stage) that organisms ensure a close genetic relatedness among their component cells. Still other theoreticians maintain that organisms have evolved means to directly "police" the selfish tendencies in order to enhance the benefits of defection from cooperation (Boyd and Richerson, 1992; Frank, 1995). In fact, this is the reason for our proposing that the escalation to a higher level of selection into a major transition of evolution was 
caused by the cascade of the immune system response brought about by the highly coordinated mitotic clones thanks to their close kinship. The instrument employed was the heritable variations in fitness produced by genes responsible for the incompatibilities between the zygote's master regulators and the invasive genomes. This proposed escalation of higher fitness values is possible with the statistical and the mathematical machine of population genetics provided by the mosaic of small populations (Wright, 1945).

\section{CELL TYPES FOR THE IMMUNE SYSTEM OF THE METAZOA}

With genomic evolution already in existence in the multicellular metazoan, the stage was set to "jump" to a new biological "revolution" in response to the selfish sequences of alien genes. To survive attacks from the outside, multicellular individuality oriented its genetic cooperation to the new level of selection through modifiers of within-organism change, including the effect of mutation rate. And, the characteristic that enabled modifiers to reduce within-organism conflict (in the midst of every parasitic crisis) had an adaptive value, which encouraged the individuality and well-being of the organism. For the organism to improve as such and to increase its chances of survival as a better biological machine where higher-order selection operates, it has to lubricate its own coherence by frustrating its "primitive" unicellular tendency towards within-organism variation and conflict. The higher-order selection resorted to further promote the differentiation of cells into cell types with properties determined by their pattern of gene activity and the proteins produced by them. Metazoan cells did what they knew best, considering that they had all the molecular equipment needed to defend them with higher-order fitness. There are several mechanisms for the maintenance and inheritance of a pattern of gene expression, which range from the continued utilization of gene regulatory proteins to the histone packing of chromatin and structural modification of DNA (see Dillon and Grosveld, 1994). As we said before, the adaptive mechanism for the new function was the unequal inheritance of cell fate determinants. This new adaptation led selection to establish important selective coefficients in favor of a new axis of polarity.

If all these processes occurred in the cells of metazoans with an incipient sexual cycle we have all the necessary ingredients for an early evolutionary novelty, through immune reactions seminal to incipient development, even without an adaptationist framework for each genetic step.

The different processes, today common in vertebrate molecular embryology, had other functions in soft-bodied, multicellular Vendian-Cambrian-Proterozoic metazoans. What later became luxury genes exclusively used for development was just a part of the silent genome, employed when situations demanded it.

\section{JUNK DNA INCREASED NON-CODING DIGITAL INFORMATION}

When parasitic genomes were not stopped at the level of the zygote's master-regulator incompatibility, then the chromosome, as a nuclear compartment, could have acted cooperatively with its previous adaptive role, converting invasive sequences into retrotransposons. Is this the inaugural entrance of Alu's into metazoan genomes? Can this be the reason for the presence of the selfish gene as the ultimate parasite (Orgel and Crick, 1980; Doolittle and Sapienza, 1980)? In order to evolve toward a nuclear compartmentalization, selection had to 
work on the variability of the effects of cluster genes with topological meaning (McClintock, 1951). This is in line with the selection theory for gene clustering (Demerec and Hartman, 1959) and for our present need for a long evolutionary history for L1 retrotransposons as well as for the widespread presence of reverse transcriptase genes (which are useless). From the relentless attack of parasites in the Proterozoic (or early Cambrian), metazoan organisms originated the fantastic amount of "junk" DNA; products of sequences rendered innocuous to the normal genome. This innocuousness is, after all, an adaptive boundary. Gradual Darwinian selection acting on the genetic pool of the individuals subjected to the parasitism (molecular copula) resulted in an adaptive boundary when the alleles did not respond to the challenge. As we insisted before (p. 3, 4), an almost isolated mosaic of small populations are indispensable to the population genetics scenario of this theory (Wright, 1945 and others). In fact, we end up with sexual isolation as a by-product of adaptation (Hoenigsberg et al., 1988). It might be useful at this point to recall the synthetic theory of Dobzhansky, Simpson, Mayr and Huxley, advocated for divergences between populations that are the result of the accumulation of point mutations, which by selection produce a gradual sequential adaptation to local circumstances and eventually selectively separate populations from their ancestors. When isolation is not complete, selection could work to perfect the splitting mechanism (Dobzhansky, 1951).

Thus, the metazoan genome had recourse to processes, which render potentially unfit codes innocuous. Our argument is that the inoculation of DNA by a foreign molecular infix had to be engulfed by the host in order to disarticulate the invasion and make it harmless, and at the same time enrich the host's genome. The host's reaction proved to be a new leap in biological innovation, a true revolution in immunological response and survival. Maybe foreign invasions of other unprepared metazoans, whether in the past or present, do not generate any sort of creative revolution. But this one did because the host (in terms of its cell biology, both internally and between cells) was molecularly ready to engulf and utilize it as a new tool for survival! The individuals reacted selectively with all the allelic combinations of the gene pool.

The price to pay for selection and adaptation for acquiring a workable metazoan development increasing through molecular parasites that invaded multicellular metazoan individuals in the Vendian era was increasing digital information.

\section{OUR THEORY PREDICTS THAT THE ORIGINAL MULTICELLULAR METAZOAN THAT INITIATED DEVELOPMENT HAD TO HAVE A REVERSIBLE NUCLEUS}

Developmental and evolutionary biology should go hand in hand to uncover the biological machinery that places the constancy of molecular phylogeny at the center of Haeckelian homologies. Whereas studies of embryology are largely based on experiments using model organisms, evolutionary biology uses historical events to describe and investigate explanations of past patterns of diversity and change. The changes in development are due to changes in gene signaling that control cell behavior in the embryo. Our starting point is the idea that, despite different modes of very early development, all vertebrate embryos go through a surprisingly similar phylotypic stage, only to differentiate later on; and for that to be able to respond universally (as in stem cells), nuclei have been non-irreversible!

We assert that development originated in the period prior to the amazing Vendian, in response to the penetration of a multicellular metazoan by a parasite. As we said in the previ- 
ous paragraph, our theory predicts the non-irreversibility of the nucleus in metazoans during the course of their development. From the previous statement, it follows that in opposition to single natural (da natura) origins, metazoans initiate cell cycles as fortuitous mechanisms, which respond to many interacting contingent (da contingere) signals, such as cell-cell and cell-matrix contacts, and ones having to do with nutrition, growth factors, hormones, and even distant cells (Murray and Hunt, 1993). On the other hand, single-celled organisms divide primarily in response to simple nutritional signals, in many directions according to circumstantial factors (conjunctus), i.e., according to fortuitus occasions. Thus, transcription reactions, RNA splicing, epistatic control, translation, pleiotropic effects, protein activation, protein degradation, and future activations are, in almost every case, phenomena that take place in conjunctus with many different occasions. But, these reactions are not unpredictable because they do not fluctuate at random!

Thus, the initial penetration of foreign DNA was the seed that began developmental evolution in the multicellular metazoan!

Is there an unknown stage in the major transition of the evolution of sexual premetazoan organisms that required "unleashing" a phylotypic embryonic momentum before proceeding to future stages of development? Is the cloning of cells with identical genomes, whose patterns of gene activity are still reversible in the mitosis of transplanted nuclei taken from cells of the blastula, in enucleated eggs with just a cytoplasmic arrangement, telling us something of the past totipotency of genes that had not "made up their minds" about what cellular differentiation is? The DNA of such an "undecided molecular organism" really belongs to an undifferentiated stage in the past when Darwinian selection had not yet taken its pick through competition and where a higher level of selection through cooperation had not set in! Is the past still in our genome? We have a parasitic genome plus the metazoan DNA's response to the early intromission. The non-irreversibility of the cell nucleus, which operates today, is a vestige of the ancient metazoan-host response to "pretentious" genomes' attempt to govern the host's metabolism for its own survival. The cell nucleus "remembers" it by keeping "open" a mechanism, which is ready to create new cell types and cell behaviors to ensure its own survival. To be able to go back, to start with another cell type, is a plan B in early development! The cell nucleus "remembers" because its machinery has resulted from conventional natural selection.

Have we realized that we may be dealing with the kind of parasitic control whereby, as in neurobiology, certain parasites control the brain of the host? We already know that bacteria, protozoa and viruses have astonishingly sophisticated ways of using animal bodies for their own life cycles. They hijack our cells, our energy and even our behavior so they can thrive. The most fierce, fiendish and "diabolical" behavior they have evolved is their ability to change a host's behavior for their own ends. There are textbook examples that involve ectoparasites, which establish complete colonies on the surface of the body. Mites of the genus Antennophorus ride on the back of ants and, with a dazzling cunning, can produce a reflex that culminates in the ant's disgorging food for the mite to feed on by stroking its mouthparts. For amazing feats that include fatal attractions, see Berdoy et al. (2000). For a general review, we recommend a more recent and thorough work (Moore, 2002).

The only difference with our theory is that we are proposing that the invasive parasite in the early metazoan body governed cell behavior for its own benefit but selection of the individual later took over by gradually "creating" an immune reaction that culminated in development. What the early metazoan had to do in the new level of selection was to experiment with a de novo library synthesis, which allowed for a true "rational" design, that was based on the available information of the proteins, which permitted maximum flexibility in their cooperative efforts. 


\section{COOPERATION AS AN ORGANIC ADAPTATION}

Early experiments in development, starting with unicellular life forms, incorporated elements of cell biology and behavior that were inherent to certain properties of life. What we contemplate in the hierarchy of life are subtle gradual levels of complex interactions, one nested into another, as genes, cells, organisms, species and communities, which reveal that each level of life has a natural and commensurate capacity to function and to improve in units of selection during evolution and through it to adapt to unavoidable change. We call this commensurate capacity to generate change evolvability (Hoenigsberg, 2002). Although phylogenetic analysis of unicellular choanoflagellate and extant metazoan relationships, based on small subunit rDNA sequences, have produced conflicting results, the discovery of a receptor tyrosine kinase (with extracellular domain quite similar to sponges and humans) in Monosiga brevicollis (King and Carroll, 2001) is highly significant to the evolution of early unicellular molecular signaling. Thus, the ability to receive and to incorporate signals suggests an early evolutionary capability of a unicellular metazoan (such as choanoflagellates) to respond to parasitic invasions with an appropriate toolkit. The genetic organization of great complexity, which unfolded from relatively simple phenomena, was invested with an enormous evolutionary potential, thanks to the molecular toolkit already present in basal bilaterians (Hoenigsberg et al., 2008). In keeping with analyses of small subunit rDNA, which have also suggested that a group of parasitic protists, the Mesomycetozoa, clusters with choanoflagellates and Metazoa on the eukaryotic evolutionary tree (Ragan et al., 1996; Medina et al., 2001), there is sufficient theoretical logic to speculate that unicellular choanoflagellates could have been within the ecological milieu of parasites (such as protists).

In the previous lower evolutionary level, the evolution of multicellularity had all the ingredients of evolvability that launched concomitant with the heritability of fitness, a new higher level individual. However, in explaining the transition from a single cell to a multicellular organism with development and sex, one has to recognize that evolvability has a history, and by doing so, one must underscore the importance of the many cellular and developmental processes that are probably a function of the history of the lineage. Therefore, evolvability includes not only the capacity to change and the unit of selection's response to immediate selective conditions, but also the capacity of previous changes to change once more. The continuous struggle to survive against parasites, predators, changing conditions and sexual competition, force organisms to respond in an adaptable way to stress and changes (Williams, 1966; Van Valen, 1973). This is all pretty much in keeping with conventional Darwinism. It helps biology, among other things, to visualize the general phenotype of animal and plants as they change within lineages. In this theory, we use the same general criterion of Darwinism but applying it to the overall design of the last common embryonic development. Cooperation in molecular biology is just another adaptive mechanism of the gene pool of parasitic invasion. With protein innovation and the cascade of events that gradually led the way in orthoselection, it produced the new individual as the final unit of selection and adaptation. Multilevel selection is the instrument that pushes the transition up the ladder. In this instance, from cell to multicellular biology and behavior, cooperation is necessary for the emergence of selection in so far as it trades fitness of the lower level for increased fitness at the higher level of group cooperation (see Oster and Wilson, 1978; Packer et al., 1991; Maynard Smith and Szathmáry, 1995; Michod, 1999a,b; Nunney, 1999). The one hundred percent relatedness of the cells 
(=clones) of an individual constitutes the glue between the immediate interests of the cell and the long-term interests of the individual. However, the conflict between short-term and longterm selection persists.

\section{COMPLEXITY AND STABILITY}

Irrespective of the origin of archaebacteria, prokaryotes and eukaryotes, the fact is that the lineage that lost the cell wall not only had to experiment with new feeding habits (from phagocytosis to the existing mechanism of cytoskeleton and endoplasmic reticulum) but also had to evolve the most amazing biological revolution that permitted eukaryotes to have many different kinds of membranes with many different kinds of jobs. Presumably, two lineages derived from the catastrophic loss of the cell wall: one, the archaebacteria, developed a rigid cell membrane (using novel membrane-forming molecules); the other, the eukaryote, evolved an internal molecular cytoskeleton that maintained the cell in place. Actin filaments and microtubules did the job, because they resist pulling forces and the compression and shearing forces, respectively. We do not wish to exaggerate by positing that this change involving digital information is the most important revolution of cell biology! Thanks to these new constructions, all the remaining important changes were possible for our ancestors. Even modern cell division owes its precision to this magnificent feat. Protein molecules can change their shape and exert mechanochemical activity by converting chemistry into mechanical motion thanks to actin filaments and the other molecules that came to be.

The membrane revolution that took place in our ancestors ended up in the multifunctional cytoskeleton. Among the many things that our proposed Vendian ancestor can do, one in particular has to do with gene regulation: the translational machinery does not automatically follow transcription, i.e., protein regulation and eventual production of variability can also be controlled at later stages in gene expression. Parasitic invasion found the modern-coding gene that comprised a stretch of DNA containing a coding region with instructions for elaborating the right protein, adjacent control regions, promoter and enhancer for the genetic switch to carry on.

All you needed to have from the Vendian-Cambrian was the genetic toolkit, the ecological conditions and the genetic environment to carry on. The timing of gene expression and protein synthesis from the beginning of the Metazoa's incipient development must have been the central mechanisms by which selection differentiated evolvability for that circumstance. The homology of developmental mechanisms at the cellular and molecular level among distantly related organisms constitutes an evolutionary indication that the workings of the early metazoan's genome and natural selection responded with precise evolvability to circumvent the theoretical conflict between physiological stability and change by controlling cellular behavior through spatial pattern and timing. The expression of developmental genes and the epistatic manner in which they control target genes permit action now or in the future, in circumstances that aborted simple competition while adopting cooperation situated on a higher level of selection and fitness.

The timing of gene expression in the early metazoan genome was constructed as fortuitus conjunctus in response to the "danger" of the force of DNA intromission. It was natural selection for a premature immune response to molecular invasion. The immediate result had to be a mitotic cycle with aggregates of cells that evolved into an articulated system of several signals and adhesion protein families (such as receptor tyrosine kinases and cadherins). Further 
natural selection, acting on variable interactions of spatial patterns, life cycles, specializations, and complex physiology, took a complex embryonic route of cooperative actions that only in this manner could secure multicellular individuality in the embryo. The main actor in the immunological change provoked was not the relatively modest parasitic genome, which had been introduced, but the far-reaching "plastic" and creative fortuitus conjunctus mechanism produced by natural selection that gradually made possible complex networks that diminished detrimental mutational load and at the same time generated new viable molecular responses that gained access to individuality. There was nothing to revoke for there was nothing before. This point needs clarification. We would like to emphasize that "far-reaching plasticity" was not natural selection's direct function; it was eventually the result.

To evolve a coordinated structure (Kaiser, 2001) it was imperative to force cellular behavior to abandon its previous selfish condition (negentropy) in which genes are selectively rewarded in their out right competition. To that end, further increments in fitness could have been easily made through genetic clones of mitotic cells by putting forward alternative alleles of innovative protein families, which produce an adaptation of aggregates (Hoenigsberg et al., 2008), that gradually develop the much needed coordination.

We believe that the statistical result from what happened to the workings of natural selection's first unit (the individual cell) is what we see as transition to more individuality through more genetic complexity. It is likely that fitness increased with deferred gene expression as exemplified by having a promoter region as the site at which RNA polymerase binds and starts transcribing, and the enhancer region thousands of base pairs distant from the promoter.

We have left unanswered the question of stability. Physiological stability need not be concomitant with genetic stability. Archean prokaryotes appeared as generalists with no visible changes in their physiology, which permitted them to deal with widely varying environmental conditions: certainly tidal changes and osmotic fluctuations were likely to have been of daily occurrence. Through genetic redundancy, robustness and other genetic mechanisms, such as changing linkage between genotypic states, organisms can theoretically support the ruggedness of the fitness landscape (Perelson and Kauffman, 1991, cited by Gerhart and Kirchner, 1997, p. 586) through organic adaptation. Conrad (1990) considers three principles of organizations upon which groups of cells are constructed that do not affect evolvability. Compartmentalization allows some aspect of the organism to evolve new structures without exposing other mechanisms. The cell types in vertebrates can be considered an example of compartmentalization of the cell's repertoire of gene expression. There is also genomic compartmentalization within the cell, which includes enhancing regions, gene duplication and alternative splicing control. We discuss some of this here.

Not only can gene action be deferred in the manner just described, but proteins, once produced, may still not be able to function. This is very convenient for the "war" with the parasites. Apparently, this post-translational modification occurs before acquiring biological meaning in the cell, but in fact, it is a phenomenon invested with enormous potential in complexity.

\section{INVASION AND EVOLVABILITY}

We think that there is a clue in gene activity that supports the idea of an externalgenomic invasion followed by the subsequent immunological response without compromising 
evolvability. If there had not been outside help (parasitic invasion) in metazoan development, gene action would have not had an apparent "commission" to fulfill and a "harvest" of highest fitness in individuality "to gather" (multicellular evolvability). As we said before, today's apparent "amazing plasticity" is just a statistical result of the successful gradual local selection, at that time (and space). The response to invasion resulted in the machinery of evolution to secure survival through conventional selection on alternative alleles of genes in the gene pool coding for innovative signaling proteins to respond to the immediate circumstances rather than to future needs! Some biologists believe that there was a seemingly higher organismic "mission", to accomplish, at the highest level of selection in the acquisition of metazoan development. As we see it, the result was: differential evolvability, which is the same old organic adaptation. Our quotations in this paragraph have a conceptual meaning, to say that there was no teleological position to express: natural selection and its product-adaptation (organic not biotic adaptation, mind you) do not express anything because there was a better future to which to travel. Of course not, but energy saving (better fitness) through cooperation in complexity, as we have tried to express in this paper, did result in a mechanism that mimics "a wonderful road ahead".

\section{THE PRECAMBRIAN REVOLUTION WAS NOT A SIMPLE ACCRETION OF PREVIOUS COMPLEXITY}

The question we need to ask now is why change is avoided in many cellular processes, notwithstanding the fact that such processes were responsible for profound divergences in phyla such as arthropods, annelids, chordates, nematodes, and mollusks among many more! The problem of conservation in protein function and in meaningful amino acid sequences, which do not reflect phylogenetic divergence, and therefore adaptive changes, is one of the great questions for biological evolution through Darwinian natural selection. It seems as though the great metazoan spatial organization, and the cell type specialization in development that contributed to the whole body plan, was accomplished with elements that were not meant to change (i.e., secondary and tertiary protein structure), such as protein conservation, the same molecular signals and physiological stability. At what point in the Precambrian period did robust and conserved processes at the cellular level begin to contribute to diversification? From a common ancestor, an incredible explosion of new spatial organizations occurred, using the conserved processes, even before the Vendian, to different ends.

Metazoan animals appeared with $10^{3}$ to $10^{15}$ cells in some estimates, from 30 to some 300 cell types, and a wide variety of tissues and organs in an incredibly complex organic machine. These are the highly sophisticated creatures that started as single cellular types: prokaryotes and eukaryotic cells, which did not control the environment around them but evolved in response to it (the great number of individuals in a bacterial culture with practically no possibility of survival outside the emergence of new mutants is a vestige of such passive dependence on the environment). On the other hand, the cells of multicellular eukaryotes can largely produce their own intercellular conditions and actually respond to these as they control the external environment. The new level of organization demanded a new level of selection (Hoenigsberg, 2001, 2003a) with cooperation instead of Darwinian competition. With this new level of selection, the Metazoa with internal 
clones, with cycles of mitotic divisions and cell types in specific spatial arrangements that are stable and reproducible within controlled development and life cycles "prepared" themselves for a new evolvability. The secondary byproduct of the new level of selection was the elaboration of a nonlethal phenotypic change that could respond with a conserved physiology in evolution: even in the course of mutational change (we include chromosomal aberrations and intron shifts as mutational strategies).

The fingerprints of selection include a Precambrian challenge to the cell genome and biology. Precambrian multicellularity superficially appears to be just an accrual of complexity among eukaryotic cells. However, if we acknowledge the immunological response to parasitic intromission, what came later, in terms of opportunities for evolution, suggests that this response ranks very high in evolutionary importance. At this point, we must repeat that whereas single-celled eukaryotes had almost no specific control over their environment and evolved mostly thanks to random mutations, the cells of multicellular eukaryotes created their own intercellular conditions and their own cell biology and behavior, just as they created their own response to the external environment.

After repeated ecological turmoil in the Vendian, where most prokaryotes and unprepared unicellular eukaryotes very likely perished, our multicellular candidate found itself facing a period of calmness, with a new kind of selection and organic adaptation. Selective mechanisms acting on relatively uniform conditions can predict future gene pools from the present ones. The reliability of the ecological conditions of the parental generation as a basis for predicting the conditions to be faced by the offspring is of great value to predict the distribution of the genotypes and the next generation's probable frequencies. Moreover, it can also be useful in explaining the incidences of the various kinds of restrictions on linkage and on recombination in sexual processes (see Williams, 1966). Such new conditions were useful in differentiating various cell types through a special spatial arrangement that results from cellular movements and intracellular protein signals.

In general, complex combinations of transcription factors control cell differentiation (Winston and Hunter, 1996; Mannervik et al., 1999; Struhl, 1999; Naya and Olson, 1999; Scully et al., 2000; Wolpert, et al., 2002; c.f., Latchman, 2008, among other experts). Some cells, such as muscle cells, initiate the differentiation program with the expression of genes such as myoD. The protein products of the myoD gene family are themselves controlled by external signals and other protein interactions.

As far as predicting the genotypic state (heterozygous or homozygous) of each cellular stage for the regulation of transcriptions even during relatively uniform ecological conditions, it is not possible because the conditions in the parental generation as a basis for predicting the conditions to be faced by the offspring will be difficult. Especially during development, often each cellular population will demand special ecological and somatic environments. Therefore, to explain the extent to which deterministic or dispersive phenomena prevail in each particular phase of the cell life cycle, which will in turn explain the incidence of various kinds of restrictions on recombination, is bound to be a colossally improbable task. Population genetics does not abound in explanations as to what is the role of linkage in regulating recombination, and crossover rates are themselves liable to modification by natural selection (Kimura, 1956). During development, cellular stability is likely to demand a homeostatic mechanism. Indeed parsimony advises to expect facultative adaptation in contingent reactions for individual survival. 


\section{THERE ARE THREE MORE INESCAPABLE PREDICTIONS}

1. The capacity of the metazoan cell to create its own conditions (automation) resulted from a reaction norm caused by the entrance of a new genome ready to subvert the internal stability of the ancient eukaryotic cell. The immunological answer to repeated invasions was the trigger mechanism for cellular automation. Cellular automation is the new attribute in the new level of selection. If you have followed us, what we ended up with is the final organic adaptation. 2. The new metazoan that emerged from a unicellular ancestor (such as the choanoflagellate) must have had in its biochemical toolkit (besides the molecular and the genetic environment already discussed in previous pages) the molecular repertoire responsible for asymmetric segregation in order to be able to establish the axis of polarity to orient the mitotic spindle and the proteins [such as HAM, i.e., Hsn (hermaphrodite-specific-neuron)-abnormalmigration] to localize cell fate determinants on the side of the cell. Cell fates must result from automation. And, asymmetric cell division necessarily follows cytoskeleton-protein determination and regulation. 3. Therefore, we predict that some primitive multicellular metazoan should have had a conserved general mechanism for asymmetric cell division: Par-proteins that polarize the cell cortex; the intracellular G-proteins (and the other molecules in the chain including protein kinase $\mathrm{C}$ ) to direct segregation and movement of cytoskeleton construction are indispensable elements for building cell types in the last common embryo.

\section{ACKNOWLEDGMENTS}

The authors are grateful to two anonymous reviewers for their valuable ideas and concepts on microbial evolution. However, the first author considers them much too conservative for his taste. In fact, both miss the point! In a recent workshop, well-known biologists attending the meeting insisted on an evolutionary mechanism capable, in the evolution of the trp locus of E. coli, of foresight! The Yanofsky group's amazing discovery of regulation of transcription termination/antitermination, transcription attenuation (Yanofsky, 2000), was used as an example of this so-called foresight. In our view this is absurd: we believe such views emerge from thinking that groups (and even populations), can be units of selection. Finally, our heartfelt appreciation goes to Dr. Dinkar Sahal of the International Centre for Genetic Engineering and Biotechnology in New Delhi, India, for his careful reading and generous suggestions that certainly improved the text in many ways.

\section{REFERENCES}

Adoutte A, Balavoine G, Lartillot N, Lespinet O, et al. (2000). The new animal phylogeny: reliability and implications. Proc. Natl. Acad. Sci. U. S. A. 97: 4453-4456.

Aguinaldo AM, Turbeville JM, Linford LS, Rivera MC, et al. (1997). Evidence for a clade of nematodes, arthropods and other moulting animals. Nature 387: 489-493.

Bavestrello G, Benati U, Calcinai B, Cattaneo-Vitelli R, et al. (1998). Body polarity and mineral selectivity in the demosponge Chondrosia reniformis. Biol. Bull. 195: 120-125.

Berdoy M, Webster JP and Macdonald DW (2000). Fatal attraction in rats infected with Toxoplasma gondii. Proc. Biol. Sci. 267: 1591-1594.

Borchiellini C, Manuel M, Baker TA, Boury-Esnault N, et al. (2001). Sponge paraphyly and the origin of Metazoa. $J$. Evol. Biol. 14: 171-179.

Boyd R and Richerson PJ (1992). Punishment allows the evolution of cooperation (or anything else) in sizable groups. 
Ethol. Sociobiol. 13: 171-195.

Conrad M (1990). The geometry of evolution. Biosystems 24: 61-81.

Conway Morris S (1993). Ediacaran-like fossils in Cambrian Burgess Shale-type faunas of North America. Palaeontology 36: 593-635.

Corliss JB, Baross JA and Hoffmann SE (1981). A hypothesis concerning the relationship between submarine hot springs and the origin of life on Earth. Oceanol. Acta 4: 59-69.

Darwin CR (1859). On the Origin of Species. John Murray, London.

Demerec M and Hartman ZE (1959). Complex loci in microorganisms. Annu. Rev. Microbiol. 13: 377-406.

Dillon N and Grosveld F (1994). Chromatin domains as potential units of eukaryotic gene function. Curr. Opin. Genet. Dev. 4: 260-264.

Dobzhansky T (1951). Genetics and the Origin of Species. Columbia University Press, New York.

Dobzhansky T (1970). Genetics of the Evolutionary Process. Columbia University Press, New York.

Doolittle WF and Sapienza C (1980). Selfish genes, the phenotype paradigm and genome evolution. Nature 284: 601-603.

Eigen M (1971). Selforganization of matter and the evolution of biological macromolecules. Naturwissenschaften 58: 465-523.

Eigen M and Schuster P (1979). The Hypercycle, a Principle of Natural Self-Organization. Springer-Verlag, Berlin.

Erwin DH (1999). The origin of body plans. Am. Zool. 39: 617-629.

Erwin DH and Davidson EH (2002). The last common bilaterian ancestor. Development 129: 3021-3032.

Frank SA (1995). Mutual policing and repression of competition in the evolution of cooperative groups. Nature 377: 520-522.

Gánti T (1971). Az Élet Princípuma (The Principle of Life). Gondolat, Budapest.

Gánti T (1984a). Coupling of autocatalytic cycles as a possible explanation of chemical oscillators. Reac. Kinet. Catal. Lett. 24: 197-202.

Gánti T (1984b). Chemoton Theory. Vol. I. Theoretical Foundation of Fluid Machineries (in Hungarian). OMIKK, Budapest.

Gerhart J and Kirchner M (1997). Cells, Embryos and Evolution. Blackwell Science, Malden.

Griesemer JR (2003). The Philosophical Significance of Ganti’s Work. In: The Principles of Life (Gánti T, ed.). Oxford University Press, New York, 169-185.

Halanych KM, Bacheller JD, Aguinaldo AM, Liva SM, et al. (1995). Evidence from 18S ribosomal DNA that the lophophorates are protostome animals. Science 267: 1641-1643.

Hoenigsberg HF (2001). Theoretical evolution: the future of selection biology international. Int. Union Biol. Sci. (IUBS) 41: 75-80.

Hoenigsberg H (2002). The future of selection: individuality, the twin legacies of Lamarck \& Darwin. Genet. Mol. Res. 1: 39-50.

Hoenigsberg H (2003a). Cell biology, molecular embryology, Lamarckian and Darwinian selection as evolvability. Genet. Mol. Res. 2: 7-28.

Hoenigsberg H (2003b). Non-Darwinian and Darwinian prokaryotic and eukaryotic evolution - an enigma in cell biology conservation. Genet. Mol. Res. 2: 279-287.

Hoenigsberg HF (2007). From geochemistry and biochemistry to prebiotic evolution...we necessarily enter into Ganti's fluid automata. Genet. Mol. Res. 6: 258-273.

Hoenigsberg HF and Rodriguez J (1987). Population genetics in the American Tropics XXIX. Considerations of heterozygosity after bottlenecks utilizing Drosophila pseudoobscura from Colombia as the likely populational model. Evol. Biol. 1: 25-43.

Hoenigsberg HF, Ordoñez-Varela M, Montaño A and Bustos E (1988). Population genetics in the American tropics. XXXV. The pattern of genetic variation in marginal and isolated Drosophila pseudoobscura from Colombia. Evol. Biol. 2: 7-34.

Hoenigsberg HF, Tijaro MH and Sanabria C (2008). From unicellularity to multicellularity - molecular speculations about early animal evolution. Genet. Mol. Res. 7: 50-59.

Kaiser D (2001). Building a multicellular organism. Annu. Rev. Genet. 35: 103-123.

Kerr B, Godfrey-Smith P and Feldman MW (2004). What is altruism? Trends Ecol. Evol. 19: 135-140.

Kimura M (1956). Rules for testing stability of a selective polymorphism. Proc. Natl. Acad. Sci. U. S. A. 42: 336-340.

King N (2004). The unicellular ancestry of animal development. Dev. Cell 7: 313-325.

King N and Carroll SB (2001). A receptor tyrosine kinase from choanoflagellates: molecular insights into early animal evolution. Proc. Natl. Acad. Sci. U.S.A. 98: 15032-15037

Latchman DS (2008). Eukaryotic Transcription Factors. 5th edn. Elsevier: Academic Press, New York.

Lewontin RC (1970). The units of selection. Annu. Rev. Ecol. Syst. 1: 1-18.

Mannervik M, Nibu Y, Zhang H and Levine M (1999). Transcriptional coregulators in development. Science 284: 606-609. 
Maynard Smith J and Szathmáry E (1995). The Major Transitions in Evolution. W.H. Freeman, San Francisco.

McClintock B (1951). Chromosome organization and genic expression. Cold Spring Harb. Symp. Quant. Biol. 16: 13-47.

Medina M, Collins AG, Silberman JD and Sogin ML (2001). Evaluating hypotheses of basal animal phylogeny using complete sequences of large and small subunit rRNA. Proc. Natl. Acad. Sci. U. S. A. 98: 9707-9712.

Michod RE (1999a). Darwinian Dynamics, Evolutionary Transitions in Fitness and Individuality. Princeton University Press, Princeton.

Michod RE (1999b). Individuality, Immortality and Sex. In: Levels of Selection in Evolution (Keller L, ed.). Princeton University Press, Princeton, 53-74.

Moore J (2002). Parasites and the Behavior of Animals. Oxford University Press, New York.

Murray A and Hunt T (1993). The Cell Cycle: An Introduction. Oxford University Press, New York.

Naya FJ and Olson E (1999). MEF2: a transcriptional target for signaling pathways controlling skeletal muscle growth and differentiation. Curr. Opin. Cell Biol. 11: 683-688.

Nunney L (1999). Lineage Selection: Natural Selection for Long-Term Benefit. In: Levels of Selection in Evolution (Keller L, ed.). Princeton University Press, Princeton, 238-252.

Orgel LE and Crick FH (1980). Selfish DNA: the ultimate parasite. Nature 284: 604-607.

Oster GF and Wilson EO (1978). Caste and Ecology in the Social Insects. Princeton University Press, Princeton.

Packer C, Gilbert DA, Pusey AE and O'Brian SJ (1991). A molecular genetic analysis of kinship and cooperation in African lions. Nature 351: 562-565.

Perelson SA and Kauffman SA (Editors) (1991). Molecular Evolution on Rugged Landscapes. Addison Wesley, Redwood City.

Ragan MA, Goggin CL, Cawthorn RJ, Cerenius L, et al. (1996). A novel clade of protistan parasites near the animal-fungal divergence. Proc. Natl. Acad. Sci. U. S. A. 93: 11907-11912.

Rodriguez J and Hoenigsberg HF (1987). Population genetics in the American Tropics XXVIII. The stepping-stone model with local extinction and recolonizations. A mathematical note on Maruyama and Kimura's 1980 work. Evol. Biol. 1: 233-272.

Scully KM, Jacobson EM, Jepsen K, Lunyak V, et al. (2000). Allosteric effects of Pit-1 DNA sites on long-term repression in cell type specification. Science 290: 1127-1131.

Struhl K (1999). Fundamentally different logic of gene regulation in eukaryotes and prokaryotes. Cell 98: 1-4.

Van Valen LM (1973). A new evolutionary law. Evol. Theor. 1: 1-30.

Watson JD, Baker TA, Bell SP, Gann A, et al. (2004). Molecular Biology of the Gene. Pearson Benjamin Cummings, CSHL Press, San Francisco.

Williams GC (1966). Adaptation and Natural Selection. Princeton University Press, Princeton.

Williams GC (1992). Natural Selection: Domains, Levels, and Challenges. Oxford University Press, New York, Oxford.

Winston LA and Hunter T (1996). Intracellular signalling: putting JAKs on the kinase MAP. Curr. Biol. 6: 668-671.

Wolpert L, Beddington R, Jessell T, Lawrence P, et al. (2002). Principles of Development. Oxford University Press Inc., New York.

Wright S (1945). Tempo and mode in evolution: a critical review. Ecology 26: 415-419.

Wright S (1978). Evolution and the Genetics of Populations. Vol. 4. Variability Within and Among Natural Populations. University of Chicago Press, Chicago.

Yanofsky C (2000). Transcription attenuation: once viewed as a novel regulatory strategy. J. Bacteriol. 182: 1-8.

Zrzavy J, Mihulka S, Kepka P, Bezdek A, et al. (1998). Phylogeny of the metazoa based on morphological and $18 \mathrm{~S}$ ribosomal DNA evidence. Cladistics 14: 249-285. 